\title{
Sonderdruck aus
}

Klassiker Auslegen Herausgegeben von Otfried Höffe

Band 43

John Locke

\section{Zwei Abhandlungen über die Regierung}

Herausgegeben von Bernd Ludwig und Michaela Rehm

ISBN 978-3-05-005076-8 E-Book: ISBN 978-3-05-006318-8

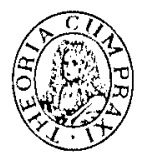

Akademie Verlag 


\section{„The A. B. C. of Politicks": Entstehungskontext und Rezeption von Lockes Zwei Abbandlungen über die Regierung}

\subsection{Der Kampf der Wbigs gegen „popery“ und „arbitrary government": Zum politischen Hintergrund}

Als "The A. B. C. of Politicks" ${ }^{\text {1 }}$ galten Lockes (1632-17042) Zwei Abbandlungen iiber dic Regiernung bereits seinen Zeitgenossen. Tatsächlich beschäftigt sich Locke hier gerade nicht mit einer abstrakten, rechtstheoretischen Begründung legitimer Herrschaft; vielmehr ist es unübersehbar, dass sein Tèxt eine Antwort auf konkrete politische Probleme seiner Epoche bieten soll. Und bei John Locke handelt es sich um einen eminent politischen Denker auch in dem Sinn, dass er nicht von einem philosophischen Elfenbeinturm herab bestimmte politische Entwicklungen als Beobachter kommentiert hätte: Als Protagonist der $W_{b i g s}{ }^{3}$ hat er sie selbst mit angestoßen und gestaltet. Er war Teil dieser politischen Bewegung, und „Two Treatises of Government was, in effect, the political manifesto of this movement". ${ }^{4}$ Mehr noch: Es ist nicht so, dass die Wligs in der politischen Philosophie Lockes die passende Doktrin gefunden und für ihre Zwecke nutzbar gemacht hätten. Diejenigen politischen Ideen, die inzwischen mit dem Namen Lockes verbunden werden, haben sich erst in der Auseinandersetzung mit den politischen Zielen der Whigs herausgebildet. Noch in seinen 1660 und 1662 entstandenen Two Tracts vertritt Locke Thesen, die seiner späteren Theorie geradewegs entgegen-

1 So äußert sich der radikale Wbig Walter Moyle um 1697 (Moyle 1727, 58).

2 Eine Kurzbiographie Lockes bietet Brandt 1988, 619-624, eine ausführliche Biographie Woolhouse 2007.

$3 \mathrm{Zu}$ den beiden politischen Hauptströmungen während der Restauration siehe Southcombe/Iapsell 2010, 48: "[...] those who believed that the greatest threat came from imminent Catholic elespotism emerged by $1680 / 1$ as ,Whigs', whilst those who dreaded Protestant sectarian zeal and social anarchy rallied to the Crown's defence as "Tories'."

4 Ashcraft 1986, 9. 
stehen; so verneint er beispielsweise ein Recht auf Selbstverteidigung der Individuen gegenüber dem Monarchen. ${ }^{5} \mathrm{Zu}$ dem im weitesten Sinne liberalen politischen Denker, wie wir ihn heute kennen, wurde Locke wohl erst durch die Begegnung mit dem Whig-Anfuihrer Shaftesbury und dessen Zirkel von 1666 an: "Shaftesbury was an ardent defender of the people's legislative power, of freely elected annual parliaments, and of limitations upon the king's prerogative, a leading advocate of toleration, vehemently anti-Catholic, and an opponent of the divine right theory of monarchy to the point of supporting armed resistance long before Locke began to write the Two Treatises". Als Sekretär und Berater von Shaftesbury war Locke unmittelbar an der Planung und Durchführung des wichtigsten $W b i g$-Projektes jener Zeit beteiligt, „the organization of a political movement to exclude James from the crown“. ${ }^{8}$ Von 1679 an versuchten die Whigs, den Bruder von König Charles II. - den Duke of York, später James II. - von der 'Thronfolge auszuschließen, weil sie befürchteten, er als Katholik werde den Katholizismus in England einführen. ${ }^{9}$ Das galt deshalb als Bedrohung, weil Katholizismus mit politischem Absolutismus identifiziert wurde, und zu der Angst vor innerstaatlichem Verlust an bürgerlichen Freiheitsrechten gesellte sich jene vor Fremdherrschaft, denn es schien nicht ausgeschlossen, dass sich ein katholischer Herrscher mit einem absolutistischen, katholischen Reich wic etwa Frankreich verbïnden oder er England sogar an eine solche Macht ausliefern würde. ${ }^{10}$ Die Whigs gewannen 1679, 1680 und 1681 die Wahlen für die Commons; drei Mal versuchten sie erfolglos, die sogenannte „Exclusion Bill“ über den Ausschluß James II. durchzusetzen; ${ }^{11}$ nach der Auflösung des „Oxford Parliament" im März 1681 durch Charles war keine weitere Einberufung des Parlaments geplant. ${ }^{12}$ Nachdem das Volk also dreimal in Folge durch die Wahl der Wljigs seinen Willen erklärt hatte, James II. nicht auf dem Thron zu akzeptieren, und dieser Wille mifachtet worden war, stand für Locke fest, dass eine Tyrannei drohte und das

5 Tuilly $1991,627$.

6 Cranston 1952, 620. Line kritische Untersuchung der historischen Zeugnisse der Beziehung zwischen Shaftesbury und Locke liefert J. R. Milton 2011. Auch J. R. Milton betont, Lockes bedeutende Werke der politischen 'Theorie wie der Essay concerning Toleration und die Tivo Tientises of Government seien während Lockes "period of [...] association with Shaftesbury" begonnen worden (ebd., 167).

7 Asheraft 1980, 42-43.

8 Ashcraft 1980, 44.

9 James II. war wohl zwischen Ende 1669) und 1672 zum Katholizismus konvertiert (Asch 2011, 92).

10 Zur antikatholischen Stimmung während der Restaurationszeit siehe Southcombe/Tapsell 2010, Kap. 5.

11 Die Forderung nach einem Ausschluss des Duke of York von der Thronfolge wurde in drei aufeinander folgenden Parlamenten vorgebracht, den sogenannten „Exclusion Parliaments“ (März bis Juli 1679, Oktober 1679 bis Januar 1681, März 1681), siche Finds 2010, Appendix 4. Der Inhalt der „Exclusion Bill“ ist abgedruckt in Browning 1953, $113 \mathrm{f}$.

12 Ashcraft 1986, 313: Nach der Auflösung des „Oxford Parliament" ,it was ,generally believed" that the king did not wish Parliament to meet and that he had no intention of calling a new one“. 
Volk sich wehren mußte: „Locke's argument in chapter thirteen of the Second Treatise [...] is especially relevant to the question of the people's right to self-defense in a situation when the legislature cannot meet or is prevented from meeting by the king [...]. This argument only made sense after the dissolution of Parliament at Oxford and the king's refusal to call for new elections." 13 Die Zavei Abhandlungen sind damit kein Pamphlet zur Legitimierung der Glorious Revolution, ${ }^{14}$ sondern ein "Exclusion tract" ${ }^{15}$ Obwohl der unmittelbare Kontext also nicht die Glorious Revolution, sondern die Exclusion Crisis war, ist offenkundig, dass sich während der Glorious Revolution jedenfalls viele der Ideen durchsetzen sollten, die Locke im Rahmen der Exclusion Crisis geäußert hatte. Denn schließlich hatte die Forderung nach einer Exklusion des Duke of York als plakative Überschrift für das viel weiter gefaßte politische Programm der Whigs gedient, das der Abwehr von "popery" und ,arbitrary government" generell gewidmet war. ${ }^{16}$ Das Projekt der „Exclusion“ sollte spektakulär scheitern; der Duke of York bestieg 1685 als James II. den Thron von England und Irland und als James VII. den Thron von Schottland. Die Amtsführung des neuen Königs zeigte jedoch rasch, dass die Befürchtungen der Whigs berechtigt gewesen waren: „King James VII and II was allegedly responsible for decisions and policies which, individually and collectively, might have been described as ,arbitrary government'. These included: the prorogation of a loyal and initially co-operative parliament, which was never to meet again [...], the dismissal of judges in the common law courts [...], the assertion of another prerogative power to dispense individuals from the law altogether" und weitere Maß3nahmen, die als Schritte in Richtung Absolutismus gedeutet werden konnten. ${ }^{17}$ Den Whigs war es zwar nicht gelungen, den Duke of York als König zu verhindern, doch die Art seiner Regentschaft ließ sich als Bestätigung dafür heranziehen, dass sie mit ihrem politischen Programm richtig lagen. Wie sollte man nun mit dem Faktum eines katholischen Königs umgehen? James II. war 51 Jahre alt, als er den 'Thron bestieg, und da die Angehörigen des Hauses Stuart nicht als langlebig galten, setzten vicle seiner politischen Gegner auf seinen frühen Tod und die Übernahme des Throns durch seine protestantische Tochter Mary. ${ }^{18}$

\section{Ashcraft 1980, 103, Fußßnote 148.}

14 Goldie/Wokler 2006, 47. Ashcraft 1986, 590 nennt die 'These von den Zwei Abbandhungen als Rechtferti gungsschriften der Glorious Revolution die "previously accepted orthodoxy".

15 Diese 'These vertreten u. a. Dunn 1984, 31; Laslett 1991; Laslett 1988, 61; Ashcraft 1980, 44.

16 Zur politischen Agenda der Wbigs siehe Glassey 2011, 223; zum ,programme of exclusion“ der Whigs als „necessary prerequisite to the success of the Revolution of 1688-89“ siche ebd. $20 \%$.

17 Glassey 2011, 210. Zu James' Versuchen einer Re-Kaxholisierung Englands siehe Southcombe/Iapsell 2010, 88 ff. Pincus 2009, Kap. 7, stellt die Standard-Interpretation von, Gelehrten in der Whig-'Tradition', "that James's irrational and un-English policies united the country against him" in Frage und prasentiert eine „revisionistische“ Lesart der Politik James': „[...] it was, above alf, James's alienation of the intolerant Anglican establishment that caused him to lose his throne" (ebd., 179).

18 Southcombe/Tapsell 2010, $87 \mathrm{ff}$. 
Die Hoffnung, dass die Regentschaft eines katholischen Königs nur ein kurzes Intermezzo bleiben würde, wurde jedoch durch die Geburt von James Francis Edward, dem Sohn James' und seiner katholischen zweiten Ehefrau Maria von Modena, erschüttert. ${ }^{19}$ James' überwiegend protestantische Untertanen waren nun mit der für sie bedrohlichen Aussicht konfrontiert, auf Dauer unter katholischer Herrschaft leben zu müssen. 1688 unternahm es James II. erneut, seine „Declaration of Indulgence“ zugunsten von Toleranz gegenüber anderen als den von der Church of England vertretenen Überzeugungen und in ihr praktizierten Riten durchzusetzen; die Erklärung sollte von jeder Kanzel des Landes herab verlesen werden. ${ }^{20}$ Damit verlangte James von den Vertretern der Church of England nichts Geringeres, als selbst das Ende ihrer Vormachtstellung zu verkïnden. ${ }^{21}$ Angesichts der wahrgenommenen Gefährdung der religiösen und politischen Freiheiten schickte eine aus Tories und Whigs bestehende Gruppe, die als ,immortal seven" berühmt werden sollte, im Juni 1688 eine Aufforderung an Wilhelm von Oranien, sich einzumischen. ${ }^{22}$ Wilhelm landete am 5. November 1688 in England; James gelang es nach einem ersten misslungenen Fluchtversuch schließlich, sich nach Frankreich abzusetzen. „[...] William's intervention was only possible because of a pre-existing crisis of confidence amongst James's subjects. That crisis was the result of the king's inadvertent success in activating powerful social, political, and religious forces which combined the rational and the irrational, the intellectual and emotional. In particular, he trespassed on noble and gentry honour codes; appeared to be governing against their interests; and seemed to be trying to force his Protestant subjects to act against the dictates of their consciences." 23

Locke hatte das Ende der Herrschaft Charles II. und die Regentschaft James' II. aus dem Exil verfolgt. Bereits nach der Entdeckung des „Rye House Plot" ${ }^{44}$ hatte Locke offenbar erkannt, dass ihm als berüchtigtem Streiter für die Sache der Whigs Gefahr drohte. Er deponierte einige seiner Papiere bei Weggefährten und begab sich schließlich 1683 nach Holland, wo sich viele der aus England gekommenen politischen Flüchtlinge

19 Southcombe/Tapsell 2010, 91.

$20 \mathrm{Im}$ April 1687 hatte James seine erste „Declaration of Indulgence" (auch: „Declaration for the Liberty of Conscience“) verkündet (Gibson 2009, 64-72); im April 1688 wurde sie erneut aufgelegt (ebd., 78-96). James erklärte die Strafgesetzgebung in kirchlichen $A$ ngelegenheiten für aufgehoben: Strafen „[...] for not coming to church, or not receiving the Sacrament, or for any other nonconformity to the religion established" wurden suspendiert (Declaration of Indulgence of King 7 ames II, April 4, 1687, zitiert nach Browning 1953, 399 f.).

21 Southcombe/Iapsell 2010, 92.

22 Pincus 2009, 261; der 'Text des Briefes ist alggedruckt in Browning 1953, 120-122.

23 Southcombe/Tapsell 2010, 96.

24 In Jahr 1683 hatten Verschwörer aus dem Kreise der Wbigs erfolglos versucht, den König und den Duke of York im „Rye House“ umzubringen (Pincus 2009, 104). 
nach James' Thronbesteigung mit Plänen für eine Invasion Englands beschäftigten. ${ }^{25}$ Welche Rolle Locke dabei gespielt hat, lässt sich nicht mit Sicherheit sagen. Richard Ashcraft geht davon aus, dass Locke aktiv in die Planungen einbezogen war; ${ }^{26}$ laut Roger Woolhouse kann ein solches Engagement nicht eindeutig nachgewiesen werden. ${ }^{27}$ Es gibt jedenfalls Belege dafür, dass Locke als ausreichend verdächtig galt, um im Exil von englischen Regierungsspionen beobachtet $z u$ werden, ${ }^{28}$ dass er mit Personen verkehrte, die an „Monmouth's Rebellion“ mitwirkten, und mit solchen, die an den Plänen für eine Invasion durch William III. beteiligt waren. ${ }^{29}$ Lockes Name stand denn auch auf einer Liste englischer Exilanten, deren Auslieferung James von der holländischen Regierung verlangte. ${ }^{30}$ Viele dieser Flüchtlinge genossen die Protektion von William und Mary von Oranien, und Locke hatte mit ihnen sogar persönlich Bekanntschaft gemacht. ${ }^{31}$ Nach Williams Landung in England galt es, seine rechtliche Position und die seiner Gemahlin Mary zu klären; zu diesem Zweck berief William das „Convention Parliament" ein, das am 22. Januar 1689 zusammentrat. Locke verfolgte diese Entwicklungen aufmerksam und äußerte Unmut über das „Parliament“, das sich seiner Meinung nach mit der Klärung der wichtigsten Frage zu viel Zeit ließ $:^{32}$ Es beschäftigte sich mit der Bildung von Ausschüssen, statt das Machtvakuum rasch zu füllen und William und Mary so schnell wie möglich die Krone anzubieten. Letzteres erfolgte schließlich am 13. Februar 1689, als das "Convention Parliament" die „Declaration of Right" verabschiedete. Dieser Erklärung zufolge hatte James durch seine Flucht nach Frankreich abgedankt, der Thron war frei und konnte mit William und Mary neu besetzt werden. ${ }^{33}$ Lockes Engagement für William und Mary war nicht unbemerkt geblieben, und

25 Zu Lockes Reaktion auf das „Rye IIouse Plot“ siehe Woolhouse 2007, 192; zur englischen Exilgeneinde in Holland siehe Pincus 2009, $324 \mathrm{f}$.

26 Ashcraft 1986, 466.

27 Woolhouse 2007, 21\%.

28 Woollouse $2007,217$.

$29 \mathrm{Zu}$ Lockes Verhälmis zu den Beteiligten an „Monmouth's Rebellion" siehe Woolhouse 2007, 217-219) und Pincus 200\%, 112: "There can be no doubt that Locke knew and socialized with many of Monmouth's supporters"; von Monmouth selbst und seinen Plänen habe Locke jedoch wenig gehalten. Zur geplanten Invasion durch William III. siehe Pincus 2009, $261 \mathrm{ff}$.

30 Woolhouse 2007, 219: „Getting wind of Monmouth's intentions, James took measures against the English fugitives sheltering in the Dutch Republic, and on I/II May, Skelton was sent a list of eighty or so people he wanted the States-General, the Dutch governing body, to expel from Holland [...]. [On] the list which he presented to the States-General he put , Mr Locke who was secretary to the Earl of Shaftesbury', as someone else who was to be ,seized and banished"."

31 Pincus 2009, 324; zu Lockes Verhältnis zu William und Mary: Fox Bourne 1876, Bd. 2, 57 f.

32 Woolhouse 2007, 262-279.

33 Woolhouse 2007, $262 \mathrm{ff}$. 
so bot William Locke schon kurz nach seiner Thronbesteigung ein wichtiges Amt an. ${ }^{34}$ Locke lehnte wegen seiner schwachen Gesundheit ab; machte aber zugleich einen Vorschlag, womit er sich stattdessen nützlich machen könnte: „If there be anything where I may flatter myself I have attained any degree of capacity to serve his Majesty, it is in some knowledge I perhaps may have in the constitutions of my country, the temper of my countrymen, and the divisions and interest among them."35 Sein Versprechen, dem König mit seinem Wissen zu dienen, wollte Locke wohl auch mit der Publikation der Zwei Abbandllungen einlösen: Die vom Manuskript der Abbandlungen übrig gebliebenen Papiere, ${ }^{36}$ so schreibt er in seinem Vorwort, „I hope are sufficient to establish the Throne of our Great Restorer, Our present King William; to make good his Title, in the Consent of the People [...]. " ${ }^{37}$ Dieser Absatz, vermutlich im August 1689 verfasst, ${ }^{38}$ wurde als Beleg dafür herangezogen, dass die Zwvei Abbandlungen zur nachträglichen Rechtfertigung der Glorious Revolution gegen James geschrieben worden waren.

\subsection{Rechtfertigung der Glorious Revolution oder „Exclusion tract"? Zur Datierung der Zwei Abbandlungen}

Die lange Zeit gängige These einer Entstehung nach der Revolution von 1688 gilt jedoch dank der Forschung von Peter Laslett als überholt. ${ }^{39}$ Inzwischen besteht im Großen und Ganzen darüber Konsens, dass beide Abbandlungen in der Zeit zwischen Lockes Rückkehr nach England Ende April 1679 und seiner Abreise nach Holland im August 1683 entstanden sein müssen. ${ }^{40}$ Strittig ist neben der genauen Datierung der Entstehungszeit auch die Reihenfolge, in der Locke die Erste und die Zweite Abbandlung verfasst hat. Peter Laslett vertritt die Auffassung, Locke habe 1679 mit der Zweiten Abbandlung begonnen; den Plan, die Erste Abbandlung zu schreiben, könne er erst nach der Publikation von Filmers Patriarcha im Winter 1679-80, nämlich Anfang 1680, gefasst haben. Richard Ashcraft dagegen stimmt mit Laslett zwar in der Ablehnung einer Datierung nach der Glorious Revolution überein, geht aber anders als Laslett davon aus, die Zweite Abbandllung sei erst nach der Auflösung des „Oxford Parliaments“ im März

34 Das genaue Angebot ist unbekannt; vermutlich handelte es sich um das Ant eines Gesandten (Woolhouse 2007, 267).

35 Zitiert nach Woolhouse 2007, 268.

36 "After his return to England, Locke found that a considerable part of the First Treatise had been lost" (Woolhouse 2007, 275).

37 Locke 1988a, Preface, 137.

38 Ebd., 137, Fußnote.

39 Laslett 1988, 59-66.

40 Milton $1995,356$. 
1681 entstanden. Die Erste Abbandlung kann ihm zufolge nicht vor April 1680 begonnen worden sein. ${ }^{41}$ Eine sichere Auskunft über die Entstehungszeit der beiden Abhandlungen ist zum gegenwärtigen Stand der Forschung nicht möglich. J. R. Milton hat die Hypothese aufgestellt, die Erste Abbandlung sei rasch nach Lockes Erwerb eines Exemplars von Filmers Patriarcha am 22.1.1680 begonnen worden, im Frühjahr oder Sommer 1680 habe Locke bereits einen Entwurf angefertigt. Die Zweite Abhandlung ist Milton zufolge nach der Ersten Abhandhung entstanden, begonnen wurde sie Ende 1680 oder im Frühsommer 1681. Demnach musste Locke die Arbeit an der Zweiten Abhandling wegen der Verhaftung Shaftesburys $1681^{42}$ unterbrechen und konnte sie erst im Februar 1682 wieder aufnehmen. Die Kapitel XVII und XIX zur Rechtfertigung von Widerstand seien wahrscheinlich in der zweiten Hälfte von 1682 verfasst worden. ${ }^{43}$ Bei allen Unklarheiten und divergierenden Forschungshypothesen lïsst sich doch zumindest mit Sicherheit festhalten, dass es sich bei der Zweiten Ablatudlung um ein "composite work" handelt, „incorporating textual strata that differ significantly in date [... $]^{\text {“. }}{ }^{44}$ Lockes auf William III. verweisendes Vorwort muss dementsprechend kein Beleg für eine Entstehung der Zwei Abbandlungen im Kontext der Glorious Revolution sein. Historischer Hintergrund der Zwei Abbandlungen, so die weitgehend einhellig vertretene Auffassung in der jüngeren Locke-Forschung, ist die „Exclusion Crisis“ ${ }^{45}$ Damit wird jedoch nicht bestritten, dass es Abschnitte in der Zweiten Abhandlung gibt, die erst kurz vor deren Veröffentlichung $1689 / 1690^{46}$ von Locke hinzugefügt wurden und die recht eindeutig Bezug auf die Ereignisse um James' Flucht und Williams Thronbesteigung nehmen. ${ }^{47}$

41 Ashcraft 1987, 286-97, 357. Ashcrafts Position wird geteilt von David Wootton (in Locke 1993a), Mark Goldie (in Locke 19931), John Marshall 1994.

$42 \mathrm{Zu}$ Shaftesburys Festnahme siehe Philip Milton 2011, bes. 237.

43 Milton 1995, $38 \%$.

44 Ebd., 388.

45 Laslett 1988, 61; diese These vertreten u. a. auch Ashcraft 1980, 44 und Dunn 1984, 31.

46 Der vollständige Titel der Erstausgabe lautet Two Treatises of Govermment. In the former the false Principles, and Foundation of Sir Robert Filmer, And bis Followers, are detected and overtbrown. The latter is an Fssay concerming the true Origimal, Extent, and End of Civil Govermment, erschienen in London, verlegt durch Awnsham Churchill. Als Erscheinungsdatum wird das Jahr 1690 genannt, in Wirklichkeit ist der Band aber 1689 publiziert worden, und zwar anonym: „Locke hat sich außer in seinem Testament vom 7. April 1704 [...] nie als Autor der "Two treatises [sic] bekannt, auch dort nicht, wo er sie als ein wichtiges Werk bezeichnet [...]“ (siehe Brandt 1988, 608; zum Publikationsdatum siehe auch Goldie/Wokler 2006, 43, zur Anonymität ebd., 47).

47 Es handelt sich vor allem um foigende Passagen der Zweiten Abhandlung: II $\$ \$ 20,171,205,21 \%, 220$. Siehe auch Woolhouse 2007, 275. 


\subsection{Reaktion auf Hobbes' Leviathan oder auf Filmers Patriarcba? Der ideengeschichtliche Hintergrund}

Dass ein inhaltlicher Schwerpunkt der Zwei Abbandlangen darauf liegt, die Illegitimität des Absolutismus nachzuweisen, wird häufig als Beleg dafür herangezogen, dass sich John Locke hier besonders gegen Thomas Hobbes richtet. Bezieht man diese These auf die von Hobbes in seiner politischen Philosophie vorgebrachten Argumente zugunsten absolutistischer Gewalt, ist sie nicht von der Hand zu weisen, lassen sich doch viele der Überlegungen Lockes fraglos zur systematischen Kritik der Hobbesschen Ideen heranziehen. Versteht man sie jedoch als auf den Autor Thomas Hobbes bezogen, lïsst sich diese These schwer belegen, weil sich Locke in den Zwei Abbandlungen nicht direkt mit seinem Werk auseinandersetzt. ${ }^{48}$ Dass die Zwei Abbandlungen für eine Leserschaft des 20. oder 21. Jahrhunderts dennoch streckenweise wie ein Kommentar zu Hobbes klingen, hat mit der erfolgreichen Rezeption des Leviatban zu tun, der aus heutiger Sicht das Werk zur Rechtfertigung des Absolutismus schlechthin zu sein scheint. Für Locke und seine Zeitgenossen war jedoch ein anderer Apologet absolutistischer Gewalt viel wichtiger, der inzwischen ironischerweise fast nur noch wegen Lockes Auseinandersetzung mit ihm bekannt ist: Robert Filmer. Weil Robert Filmers Schriften nicht annähernd an Hobbes' Werke heranreichen, was die philosophische Qualität der vorgebrachten Argumente betriff, nimmt es nicht wunder, dass Filmer heute nahezu vergessen ist, Hobbes aber als philosophischer Klassiker gilt. Zu Lockes Zeiten aber war Filmers Theorie trotz ihrer argumentativen Schwächen sozusagen zur „official state ideology “49 geworden, an der man aus politischen Gründen nicht vorbeikam, auch wenn man sie in philosophischer Hinsicht - wie Locke - nicht ernst nahm: ${ }^{50}$ „Filmer's works were among the most powerful which opposed historical Parliamentary rights to alter the course of the succession and the most powerful theoretical argument supported by clerics endorsing Charles' moves towards absolutism, especially in the years following the dissolution of the 1681 Oxford Parliament. As royalism came to be increasingly dependent upon clericalist and biblicist Filmerian patriarchalism instead of the more moderate

48 Hobbes' Leviathan findet sich in der Ausgabe von 1651 in Lockes Buchbestand, ebenso kritische Auseinandersetzungen mit Hobbes, etwa von George Lawson oder von Edward Clarendon (Harrison/Laslett 1965, 109, 155, 170). Doch Peter Laslett hat in seinen Anmerkungen zur von ihm herausgegebenen Edition der Two Treatises (Locke 1988) auf die Stellen in diesem Werk aufmerksam gemacht, die sich auf Hobbes zu beziehen scheinen, und jeweils Gründe genannt, weshalb dieser Eindruck trüge (siehe seinen Index zu „Hobbes, Thomas" und zu "Leviathan"). Laslett betont den Umstand, "that it has not been possible to find a single referenced extract from the work of Hobbes in the whole Lockeian corpus“ (Laslett 1988, 74).

49 Ashcraft 1980, 80.

50 Im Vorwort zu den Two Treatises macht Locke deutlich, was er von Filmers Werk hält: „[...] there was never so mucb glib Nonsence [sic] put togetber in well sounding English" (Locke 1988, Preface, 137 f.), und erklärt, warum er sich trotzdem mit ihm beschäftigt: "I should not speak so plainly of a Gentlentm, long since past answering, bad not the Pulpit, of late Years, publickly owned bis Doctrine, and made it the Currant Divinity of the Times" (ebd., 138). 
and constitutionalist royalism of many Restoration legists, Locke would have had good reason to oppose Filmer's arguments [...]." Locke wie auch andere politische Theoretiker seiner Zeit wie James 'Tyrell oder Algernon Sidney sahen daher die Notwendigkeit, Filmers Thesen zu widerlegen. ${ }^{51}$ Filmers Theorie „natürlicher Untertänigkeit“ war bestens zur Zementierung des politischen Status quo geeignet, verkündete Filmer doch, jeder Mensch werde in eine Herrschaftsform hineingeboren und habe nicht das Recht, diese in Frage zu stellen. In Filmers Augen ging Thomas Hobbes mit seiner Verteidigung des Absolutismus nicht weit genug, weil er Ausnahmen einräumte, was den Gehorsam gegenüber dem absolutistischen Souverän angeht: Gegen einen Tyrannen, der einem nach dem Leben trachtet, darf man sich laut Hobbes wehren - für Filmer eine gefährliche Lehre, die der Rebellion Tür und Tor öffnete. ${ }^{52}$ 'Thomas Hobbes magr schon früh als „Monster of Malmesbury“ gegolten haben. Was jedoch den Umfang der souverïnen Gewalt betrifft, geht Filmer weiter als Hobbes, und für einen Verteidiger begrenzter Staatsmacht wie Locke war er deshalb nicht nur wegen seiner Popularität unter den Mächtigen seiner Zeit, sondern auch aus inhaltlichen Gründen der wichtigere Gegner.

\subsection{Nur ein zeitgeschichtlich interessantes politisches Pamphlet?}

Die Zwei Abhandlungen sind also in unmittelbarer Reaktion auf dic politischen Geschehnisse und auf die prominente Doktrin der Zeit, Patriarcha, entstanden. Das bedeutet jedoch nicht, dass sie nur als Zeitdokument zu lesen wären, von Interesse allenfalls aus ideengeschichtlicher Perspektive. Locke antwortet auf eine bestimmte historische Situation, doch das Problem, mit dem er sich auseinandersetzt, beschränkt sich nicht auf seine Epoche: Wann und wo auch immer politische Macht missbraucht zu werden droht, sind Lockes Überlegungen aktuell - um den Machtmissbrauch zu identifizieren, ihn abzuwehren und Maßnahmen zu seiner Verhinderung zu ergreifen.

Dass die Zwei Abbandlungen nicht als politisches Pamphlet abgetan werden können, zeigt sich auch darin, dass Lockes politische Theorie auf dem soliden erkenntnistheoretischen Fundament gründet, das er im Versucb iiber den menscblicben Verstand präsentiert (1689 erschienen, in Arbeit seit $1671^{53}$ ). Wenn es keine angeborenen Ideen oder Prinzipien gibt und Erkenntnis nur durch Ėrfahrung möglich ist, wie Locke im Versucls zeigen

51 John Dunn 1984, 32 betont, Fïlmer sei zu Lockes Lebzeiten eine gängige Zielscheibe politischer Schriftsteller gewesen.

52 Filmer 1991, 219-227 und Tully 1991, 621: ,Many agreed with Filmer, especially after the failed radical Whig uprising and the Rye House Plot of 1681-3: the major tenets of natural freedom were condemned by Oxford University, and Locke's fellow revolutionary Algernon Sidney was executed for holding them."

53 Im Frühjahr 1671 verfaßte Locke einen ersten Entwurf zum 'Thema des Versucb ibber den menscllichen Ver-

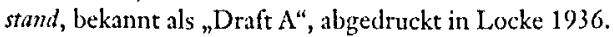


will, ergibt sich daraus eine politisch brisante Konsequenz: Das Individuum kann und soll die „Behauptungen politischer Interessenten auf keinen Vorwand hin glauben, sondern mit der Urteilskraft selbst überprüfen, z. B. Behauptungen wie diese: Der Mensch ist von Natur nicht frei, Adam war jure divino der absolute Herrscher der Welt, die heutigen Monarchen sind die legitimen Nachfolger Adams, ergo ist ihre Monarchie göttlichen Rechts und absolut." ${ }^{\text {"54 }}$ Locke entlarvt die Berufungen auf Tradition, auf angeblich von allen als selbstverständlich erkannte Wahrheiten als Instrument der Herrschaftssicherung und kritisiert, „die Menschen in ihren Parteien [zwängen] ihre Lehrsätze allen Leuten, deren sie habhaft werden können, gewaltsam [auf], ohne ihnen zu gestatten, sie auf ihre Richtigkeit oder Unrichtigkeit hin zu prüfen [...] “. ${ }^{55}$ Die in den Zwei $A b$ bandlangen geäußerte Kritik an Filmer und Konsorten ist eben nicht nur eine politisch opportune Schmähung der Gegner der Wbigs: Für Locke täuschen sie sich, weil sie ihre Argumente auf Einsichten stützen, die sie gar nicht besitzen können, weil etwa angeborene Ideen nicht existieren. Demgegenüber setzt er auf die Fähigkeiten, die seiner Auffassung zufolge jedes Individuum besitzt, die den Menschen vom Herrschaftswissen politischer oder kirchlicher Autoritäten unabhängig machen und es ihm ermöglichen, zu eigenständigen Urteilen zu gelangen. Diese „geistige Ausrüstung“ bildet die wahre Grundlage aller Erkenntnis - sogar des Wissens von der Existenz Gottes: „Sinne, Wahrnehmung, und Vernunft".56

\subsection{Zur Rezeption im 18. Jahrhundert}

Lockes Ruf als herausragender Denker gründete bis Mitte des 18. Jahrhunderts hauptsächlich auf seiner theoretischen Philosophie. Von diesem Ruhm sollten jedoch auch seine Werke zur politischen Philosophie profitieren - die Zwei Abbandlungen wurden zunächst nicht in erster Linie wegen ihres Inhalts, sondern „als Werk des berühmten Autors des "Essay" zitiert und empfohlen". 57 Die in den Zwei Abbandlungen vorgestellten Überlegungen verbreiteten sich aber trotz dieses Umwegs und beeinflussten andere politische Denker maßgeblich. ${ }^{58}$ Jean-Jacques Rousseau etwa greift in Vom Gesellschaftsvertrag (1762) Lockes Freiheitsbegriff auf und entwickelt ihn weiter. Er stimmt Locke zu, dass ein Staat, der seine Bürger zwar vor Übergriffen von außen schützt, ihnen

54 Specht 1972, 204.

55 Locke 19883, Buch IV, Kap. 3, $\$ 20$.

56 Locke 1988b, Buch IV, Kap. 10, \$1. Laut Brandt 1988, 682 wendet sich Locke damit „gegen die Legitimität eines bloss ererbten, nicht erworbenen Erkenntnis- oder Realbesitzes. Die,Gedankenarbeit' (labour of thought) ist das Gegenstïck zur materiellen $\Lambda$ rbeit bzw. zum ,ehrbaren Fleiss" (honest industry)" - für Locke ist es Faulheit, sich von anderen einfach vorsagen zu lassen, was man zu glauben und zu denken hat.

57 Brandt 1988, 704 und Dunn 1969, 79-80.

58 Zur frühen Rezeption der Abhandlungen bis 1705 siehe Pocock 1991. 
aber ihre Freiheit nimmt, illegitim ist - Freiheit im Sinne einer Freiheit vom Willen anderer ${ }^{59}$ wird von Locke und Rousseau als so zentral erachtet, dass man sie nicht abtreten könne, ohne damit auf seine Selbsterhaltung (Locke) oder gar komplett auf sein Menschsein (Rousseau) zu verzichten. ${ }^{60}$ Rousseau bedient sich sogar des Lockeschen Beispiels, um die Absurdität von Staaten aufzuzeigen, welche den Mangel an Freiheit ihrer Bürger damit rechtfertigen wollen, dass sie diesen doch immerhin ein Leben in Frieden ermöglichten: Solche Staaten funktionierten wie die Höhle des Polyphem, in der die Gefährten des Odysseus überlebten, solange sie nicht aufmuckten und sich schön still verhielten. ${ }^{61}$ Locke mag auch die erstmals von Rousseau vorgestellte Theorie konstitutioneller Volkssouveränität vorbereitet haben. Locke zufolge besitzt das Volk „Supream Power“; er beeilt sich aber hinzuzufügen, dies sei nicht im konstitutionellen Sinn zu verstehen. ${ }^{62}$ Während Locke das Volk als souverän gemäß dem Naturrecht betrachtet, ihm aber keinen institutionellen Platz in der Verfassung des Gemeinwesens einräumt, macht Rousseau deutlich, dass die von Locke übernommene Vorstellung von Freiheit nur in einer direkten Demokratie mit dem Volk als konstitutionellem Souverän denkbar ist. ${ }^{63}$

Und gerade für Denker, die sich mit ähnlichen Problemen wie Locke zur Entstehungszeit der Zwei Abbandlungen herumschlagen mussten, war dieses Werk attraktiv: Es bot nicht nur eine Theorie der Herrschaftslegitimation und -limitation, sondern auch konkrete Vorschläge zu deren Umsetzung, beispielsweise in Form einer ausgearbeiteten Lehre der Gewaltenteilung. So beriefen sich die Urheber der Amerikanischen Unabhängigkeitserklärung und der Verfassung auf Locke, ${ }^{64}$ und bei der Lektüre insbesondere von $\$ 2$ der „Declaration of Independence“ scheint offenkundig zu sein, dass ihr Lockes Theorie zugrunde gelegen haben muss: „We hold these truths to be self-evident, that all men are created equal, that they are endowed by their Creator with certain unalienable Rights, that among these are Life, Liberty, and the pursuit of Happiness. - That to secure these Rights, Governments are instituted among Men, deriving their just powers from the consent of the governed. - That whenever any Form of Government becomes destructive of these ends, it is the Right of the People to alter or to abolish it, and to institute new Government, laying its foundation, and organizing its powers in such form, as

59 Locke II \$22; Rousseau 1964, I, 8, 365.

60 Locke II, $\$ 23$; Rousseau 1964, I, 4, 356.

61 Locke II, \$228; Rousseau 1964, I, 4, 355-356.

62 ,And thus the Commumity may be said in this respect to be always the Supream Power, but not as considered under any Form of Government [...]“ (II $\$ 149$ ); vgl. auch II $\$ 168:,[\ldots]$ tho' the People cannot be fudge, so as to have by the Constitution of that Society any Superiour power [...]; yet they have, by a Law antecedent and paramount to all positive Laws of men, reserv'd that ultimate Determination to themselves [...]."

63 Rousseau 1964, I, 6-7; ebd. II, 1-2; ebd. III, 15.

64 Zum Einfluß Lockes auf die amerikanische Verfassungsdebatte: Pangle 1988 und White 1987. 
to them shall seem most likely to effect their Safety and Happiness." ${ }^{65}$ Die These einer direkten Übernahme der Auffassungen Lockes wird allerdings durch Befunde in Frage gestellt, die darauf hinweisen, dass die Zwei Abbandlungen in den Kolonien bis um 1750 kaum verbreitet waren. ${ }^{66}$ Doch selbst wenn tatsächlich nur Wenige dieses Werk persönlich gelesen haben sollten, scheinen die darin enthaltenen Ideen sich herumgesprochen und Popularität erlangt zu haben, wie folgendes Beispiel zeigen mag. Thomas Jefferson als Hauptautor der „Declaration of Independence" (1776) musste sich vorwerfen lassen, er habe diese ,von Locke abgeschrieben ${ }^{67}{ }^{67}$ Jefferson entgegnete auf diese Kritik, die ganze „Autorität" der Erklärung beruhe ,on the harmonizing sentiments of the day, whether expressed in conversation, in letters, in printed essays, or the elementary books of public right, as Aristotle, Cicero, Locke, Sidney, etc. "68 Dass die geäußerten Auffassungen nicht als originell galten, verteidigt Jefferson gerade als Stärke der „Declaration“ - diese auch auf Locke zurückgehenden Ideen waren eben nicht neu, sie gehörten zu den gängigen Überzeugungen der Zeit. Sie in ein politisches Programm wie die „Declaration of Independence" aufzunehmen, dessen Durchsetzung von einer möglichst breiten Akzeptanz in der Bevölkerung abhing, zeugt davon, wie stark die Inhalte der Zwei $A b$ bandlungen bereits Eingang in die öffentliche Meinung gefunden haben müssen. John Dunns Diagnose einer ,intellectual osmosis“, durch die Lockes Ideen absorbiert worden seien, bringt die Sache auf den Punkt: Diese Osmose habe es ermöglicht, „[to] be of Locke's party without knowing it [...]“. ${ }^{69}$ Vermutlich ist es nach wie vor der Fall, dass Locke viele Parteigänger hat, die seine Schriften gar nicht kennen.

\subsection{Politischer Liberalismus auf Grundlage des christlichen Naturrechts?}

Die überwältigende Verbreitung und Akzeptanz der Lockeschen Theorien kann man als Indiz dafür heranziehen, dass diese auch unabhängig von ihrem historischen Kontext interessieren und sie nicht nur eine Fundgrube für jene sind, die nach Lösungen für konkrete politische Probleme Ausschau halten. Auch wer nicht wie Locke und seine Mitstreiter während der Exclusion Crisis gegen Fremdbestimmung kämpfen muss und wegen der Ähnlichkeit der Ausgangslage auf die Zwei Abbandlungen zurückgreift, findet in ihnen systematisch relevante philosophische Fragen und Antworten. So fragt Locke etwa, wie politische Herrscher überhaupt Autorität beanspruchen können, und bindet

65 'Tansill 1927, 22.

66 Dunn 1969, 79 .

67 Reck 19\%1, 552.

68 Ebd.

69) Dunn 1969, 79. 
jegliche legitime Herrschaft an die Zustimmung der Bürger. Er sucht nach einem Konzept, mit dem deutlich gemacht werden kann, dass diese Zustimmung mitnichten einmal gegeben und dann für immer gültig ist, sondern konstant erneuert werden muss, und findet es im Begriff von „trust“ („Vertrauen“). Er forscht nach den Ursachen von Machtmissbrauch und überlegt, wie sich dieser verhindern lässt. Und er legt dar, dass sich der Zweck des Staates nicht darauf beschränkt, die Bürger vor gegenseitigen Übergriffen und Attacken fremder Mächte zu schützen; vielmehr erstreckt er sich auch auf den Schutz der Bürger vor dem Staat. Die Individuen werden als Träger unveräußerlicher Rechte präsentiert, Rechte, die sie sogar gewaltsam gegenüber der Staatsmacht verteidigen dürfen und sollen. „Locke ist der erste Theoretiker, der die Freiheit und rechtliche Gleichheit aller Staatsbürger miteinander identifiziert" ${ }^{\text {" }}{ }^{70}$ und all diese Elemente haben dazu beigetragen, Locke zum Referenzautor für alle zu machen, welche die Aufgabe des Staates in der Wahrung von Freiheit und Gleichheit sehen, allen voran Denker in der Tradition des politischen Liberalismus. Die „commonplace view of Locke as a founding father of liberal ideas ${ }^{471}$ muss sich jedoch einem schwerwiegenden Einwand stellen: Die materialen Aspekte der politischen Philosophie Lockes können gewiss als liberal gedeutet werden, und die Erfolgsgeschichte von Konzepten wie Eigentumsschutz oder Widerstandsrecht bestätigt die These von Locke als Ahnherren des Liberalismus. Doch diese als liberal geltenden Konzepte beruhen auf einem Fundament, das möglicherweise mit dem politischen Liberalismus konfligiert - Lockes Begründungsprogramm ist das des traditionellen christlichen Naturrechts. Der Konflikt lässt sich nicht dadurch auflösen, dass man Lockes Rekurs auf das Naturrecht als Zugeständnis an das im Christentum verwurzelte Denken des 17. Jahrhunderts deutet, oline das seine Schriften keine Chance auf Anerkennung durch seine Zeitgenossen gefunden hätten. ${ }^{72}$ Denn den Dreh- und Angelpunkt der politischen Philosophie Lockes bildet die These, niemand könne einem anderen mehr Macht übertragen, als er selbst habe, und an dieser 'These hängt der im Liberalismus geschätzte Imperativ, man dürfe sich und andere keiner willkürlichen Macht ausliefern (II $\$ 6,135$ ). Der Gesetzgeber nun aber, der dies gebietet, ist für Locke zweifellos Gott (II $\$ 135$ ). Dass man sich laut Locke zum Beispiel nicht in die Sklaverei verkaufen darf, wird nicht etwa mit einem Grundrecht auf Freiheit begründet, auf das man sich als Mensch aufgrund seines Menschseins berufen könnte. Man darf sich Locke zufolge allein deshalb nicht versklaven, weil man das Eigentum Gottes ist (II $\$ 6$ ) und somit gar „keine Gewalt über sein eigenes Leben“ (II \$23) besitzt. Will man die materialen Aspekte der politischen Philosophie Lockes übernehmen, ohne deren

70 Brandt 1973, 74.

71 Miqueu 2009, 4. Zum Lockeschen Liberalismus: Grant 1991; Strauss 1953; Macpherson 1962; Salzborn 2010.

72 Diese These vertritt z. B. Macpherson 1962, 270. 
Fundierung im christlichen Naturrecht zu akzeptieren, wird man sich eine alternative Begründung überlegen müssen. ${ }^{73}$

Jedenfalls zeigt sich am Beispiel Lockes einmal mehr, dass es philosophiegeschichtlicher Unsinn ist, eine moderne, säkulare, Autonomie befördernde politische Philosophie generalisierend gegen ihre christlichen, auf Heteronomie beruhenden Vorgängermodelle auszuspielen: Bei Locke stellt das christliche Naturrecht gerade die Bedingung der Möglichkeit seines Freiheitsbegriffes dar. Und die Freiheit, die er meint, ist die bis heute geschätzte und als modern verstandene: Freiheit von Fremdbestimmung.

\section{Literatur}

Asch, Ronald G. 2011, Die Stuarts. Geschichte einer Dynastie, München: C. H. Beck

Ashcraft, Richard 1980, The "Two Treatises" and the Exclusion Crisis: The Problem of Lockean Political

Theory as Bourgeois Ideology, in: Pocock, J. G. A./Ashcraft, Richard, John Locke. Papers read at a Clark

Library Seminar, 10 December 1977, Los Angeles: William Andrews Clark Memorial Library, 27-114

Ashcraft, Richard 1986, Revolutionary Politics and Locke's „Two Treatises of Government", Princeton:

Princeton University Press

Ashcraft, Richard 1987, Locke's „Two Treatises of Government“, London: Allen \& Unwin

Brandt, Reinhard 1973, Rousseaus Philosophie der Gesellschaft, Stuttgart: Frommann-Holzboog

Brandt, Reinhard 1988, John Locke, in: Grundrib der Geschichte der Philosophie, begründet von Friedrich

Ueberweg, 3. Bd.: Die Philosophie des 17. Jahrlunderts, hrsg. v. Jean-Pierre Schobinger, Basel, 607-713

Browning, Andrew (Hrsg.) 1953, English Historical Documents, Band 8: 1660-1714, London: Eyre \&

Spottiswoode

Cranston, Maurice 1952, The Politics of John Locke, in: History Today 2 (Sept. 1952), 619-622

Dunn, John 1969, The Politics of Locke in England and America in the Eighteenth Century, in: Yolton, John

W. (IIrsg.), John Locke: Problems and Perspectives. A Collection of New Essays, Cambridge: Cambridge

University Press 1969, 45-80

Dunn, John 1984, Locke, Oxford u. New York: Oxford University Press

Filmer, Robert 1991, Patriarcha and Other Writings, hrsg, v. Johann P. Sommerville, Cambridge: Cambridge University Press

Fox Bourne, II. R. 1876, The Life of John Locke, London: Henry S. King \& Co.

Gibson, William 200\%, James II and the Trial of the Seven Bishops, Basingstoke und New York: Palgrave Macmillan

Glassey, Lionel K. J. 2011, Shaftesbury and the Exclusion Crisis, in: Spurr, John (Hrsg.), Anthony Ashley Cooper, First Earl of Shaftesbury 1621-1683, Farnham u. a.: Ashgate, 207-232

Goldie, Mark (Hrsg.) 1999, The Reception of Locke's Politics. From the 1690s to the 1830s, 6 Bände,

London: Pickering \& Chatto und Brookfield: Ashgate

73 Waldron 2002 hält es für unmöglich, den religiösen Gehalt der politischen Philosophie auszuklammern; ähnlich Müller 2011, 230: „Eine jede Locke-Interpretation, welche sich zum Ziel setzt, dessen Theorie anschlussfähig für die gegenwärtige Debatte zu deuten, muss die tragende Rolle der religiösen Annahmen berücksichtigen. Wer dem Lockeschen Argument des natürlichen Gesetzes eine andere, säkulare Basis geben möchte, wird dieses Vorhaben kaum ohne eine Umdeutung des natürlichen Gesetzes selber ausfülren können. Wic auch immer das Resultat einer solchen Reformulierung aussehen mag, ist es kaum wahrscheinlich, dass ein derartiges Argument in ein solch umfassendes und in seinen praktischen Ergebnissen vielen Theoretikern der Gegenwart hochwillkommenes System münden kann, wie Locke es entwickelt hat." 
Goldie, Mark/Wokler, Robert (Hrsg.) 2006, The Cambridge History of Eigheenth-Century Political

Thought, Cambridge: Cambridge University Press

Grant, Ruth W. 1991, John Locke's Liberalism, Chicago: University of Chicago Press

Harrison, John/Laslett, Peter 1965, The Library of John Locke, Oxford: Oxford University Press

Hinds, Peter 2010, 'The Horrid Popish Plot'. Roger L'Estrange and the Circulation of Political Discourse in

Late Seventeenth-Century London, Oxford u. New York: Oxford University Press

Laslett, Peter 1988, Einleitung zu: John Locke, „Two Treatises of Government“, hrsg. v. Peter Laslett, Cambridge: Cambridge University Press

Laslett, Peter 1991, The English Revolution and Locke's Two Treatises of Government, in: Richard Ashcraft (Hrsg.), John Locke - Critical Assessments, Bd. 1, London u. New York: Routledge, 32-49

Locke, John 1936, An Early Draft of Locke's Essay together with Excerpts from His Journals, hrsg. v. Richard I. Aaron und Jocelyn Gibb, Oxford: Clarendon Press

Locke, John 1988a, Two Treatises of Government, hrsg. v. Peter Laslett, Cambridge: Cambridge University Press

Locke, John 1988b, Versuch über den menschlichen Verstand, 2 Bände, Hamburg: Meiner

Locke, John 1993a, Political Writings, hrsg. v. David Wootton, London und New York: Penguin Books

Locke, John 1993b, Two Treatises, hrsg. v. Mark Goldie, London: J. M. Dent

Macpherson, Crawford B. 1962, The Political Theory of Possessive Individualism. Hobbes to Locke, Oxford: Oxford University Press

Marshall, John 1994, John Locke: Resistance, Religion and Responsibility, Cambridge: Cambridge University Press

Milton, J. R. 1995, Dating Locke's „Second 'Treatise“, in: History of Political Thought 16, 356-390

Milton, J. R. 2011, The Unscholastic Statesman: Locke and the Earl of Shafteshury, in: Spurr, John (FIrsg.), Anthony Ashley Cooper, First Earl of Shaftesbury 1621-1683, Farnham u. a.: Ashgate, 153-182

Milton, Philip 2011, Shaftesbury and the Rye House Plot, in: Spurr, John (Hrsg.), Anthony Ashley Cooper, First Earl of Shaftesbury 1621-1683, Farnham u. a.: Ashgate, 233-268

Miqueu, Christophe/Chamie, Mason (Hrsg.) 2009, Locke's Political Liberty: Readings and Misreadings, Oxford: Voltaire Foundation (Studies on Voltaire and the Eighteenth Century; 4/2009)

Moyle, Walter 1727, An Essay on the Lacedxmonian Government [ca. 1697], in: Ders., The Whole Works of Walter Moyle, Esq; that were Published by Himself. To Which is Prefixed some Account of his Life and Writings, London: Knapton u. a., 49-77

Müller, Johannes W. 2011, ,Das Werk eines einzigen allmächtigen und unendlich weisen Schöpfers'. Zur religiösen Fundierung der Staatsphilosophie John Lockes, in: Politisches Denken. Jahrbuch 2011, hrsg. v. Volker Gerhardt/Reinhard Meliring/Henning Ottmann/Martyn P. Thomson/Barbara Zelinpfennig, Berlin: Duncker \& Humblot, 207-234

Pangle, Thomas L. 1988, The Spirit of Modern Republicanism, Chicago: University of Chicago Press Pincus, Steve 2009, 1688. The First Modern Revolution, New Haven und London: Yale University Press Pocock, J. G. A. 1991, Negative and Positive Aspects of Locke's Place in Eighteenth-Century Discourse, in:

M. P. Thompson (Hrsg.), Joln Locke and Immanuel Kant. Historical Reception and Contemporary Relevance, Berlin: Duncker \& Humblot 1991, 45-61

Reck, Andrew J. 1991, The Enlightenment in American Law II: 'The Constitution, in: Review of Metaphysics $44,729-754$

Rousseau, Jean-Jacques 1964, Du contract social, in: Ders., Ceuvres complètes, hrsg. v. Bernard Gagnebin u. Marcel Raymond, Bd. 3, Paris: Gallimard (Bibliothèque de la Pléiade)

Salzborn, Samuel (Hrsg.) 2010, Der Staat des Liberalismus. Die liberale Statstheorie von John Locke, Baden-Baden: Nomos

Southcombe, George/Iapsell, Grant 2010, Restoration Politics, Religion and Culture. Britain and Ireland, 1660-1714, Basingstoke: Palgrave Macmillan

Specht, Rainer 1972, Innovation und Folgelast. Beispiele aus der neueren Philosophie- und

Wissenschaftsgeschichte, Stuttgart-Bad Cannstatt: Frommann-Holzboog (problemata; 12) 
Strauss, Leo 1953, Natural Right and History, Chicago: University of Chicago Press

'Tansill, Charles C. 1927, Documents Illustrative of the Formation of the Union of the American States, Washington, D. C.: Government Printing Office

'Tuckness, Alex 1999, The Coherence of a Mind: Jolnn Locke and the Law of Nature, in: Journal of the History of Philosophy 37, 73-90

'Tully, James 1991, Locke, in: Burns, J. H. mit Goldie, Mark (Hrsg.), The Cambridge History of Political Thought 1450-1700, Cambridge u. a.: Cambridge University Press, 616-656

Waldron, Jeremy 2002, God, Locke, and Equality. Christian Foundations in Locke's Political Thought, Cambridge: Cambridge University Press

White, Morton 1987, Philosophy, „The Federalist“ and the Constitution, New York: Oxford University Press Woolhouse, Roger 2007, Locke. A Biography, Cambridge: Cambridge University Press 2007 